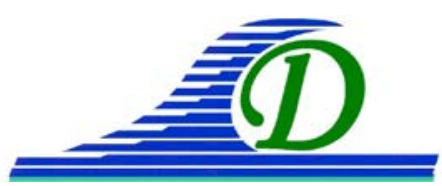
XIII İmes Journées Nationales Génie Côtier - Génie Civil Dunkerque, 2-4 juillet 2014

DOI:10.5150/jngcgc.2014.089 @ Editions Paralia CFL

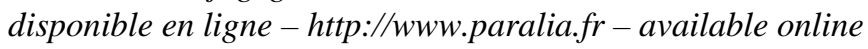

\title{
Restauration écologique d'un marais intertidal : exemple de la renaturation par dépoldérisation de l'île Nouvelle (estuaire de la Gironde, Aquitaine, France)
}

\author{
Virginie LAFON $^{1}$, Stéphane KERVELLA ${ }^{1}$, Alain LECHENE ${ }^{2}$, \\ Aldo SOTTOLICHIO ${ }^{3}$, Flore RIMOND ${ }^{2}$, Sébastien FOURCADE ${ }^{4}$, \\ Sylvain CARDONNEL ${ }^{4}$, Didier ALARD ${ }^{5}$
}

1. GEO-Transfert, UMR 5805 EPOC, al. Geoffroy St Hilaire, CS 50023, 33615 Pessac. v.lafon@epoc.u-bordeaux1.fr, s.kervella@epoc.u-bordeaux1.fr

2. IRSTEA UR EABX-Groupement de Bordeaux, 50 Av de Verdun, 33612 Cestas.

3. UMR 5805 EPOC, allée Geoffroy St Hilaire, CS 50023, 33615 Pessac cedex.

4. Département de la Gironde, 1 Esp. Ch. de Gaulle, CS 71223, 33074 Bordeaux Cedex.

5. UMR INRA 1202 BIOGECO, Bâtiment B2, Avenue des Facultés, 33405 Talence.

\section{Résumé :}

L'île Nouvelle, dans l'estuaire de la Gironde, fait l'objet d'une politique de renaturation, fondée sur une procédure de dépoldérisation. Parfaitement maîtrisée au sud de l'île, la dépoldérisation suit un cours plus naturel au nord, suite à la rupture d'une digue accompagnée d'une submersion massive répétée depuis au rythme des marées. Un projet de recherche visant à guider les choix de gestion réunit une équipe transversale qui étudie les processus de cette dépoldérisation. Nous présentons ici les méthodes d'échantillonnage de deux des volets de l'étude consacrés à l'évolution du couvert végétal et à la dynamique sédimentaire de la partie nord de l'île. Les premiers résultats montrent un processus de transformation en quatre étapes marqué tout d'abord par l'érosion et le déboisement. L’érosion et l'envasement ensuite se côtoient, ce dernier permettant une colonisation précoce des sites où la sédimentation est la plus forte par des espèces végétales typiques des zones humides. La sédimentation reste active. Les zones intertidales se comblent petit à petit. Le couvert végétal se densifie et le sol se stabilise. Outre ces transformations morphologiques et biologiques très profondes, les impacts perçus sur la faune aquatique sont également synthétisés.

Mots-clés : Dépoldérisation, Sédiments fins, Ecosystèmes estuariens, Gestion des zones humides, Marais maritime, Télédétection spatiale, Images Pléiades.

\section{Introduction}

A l'heure actuelle, les intérêts économiques et défensifs incitant à dépoldériser semblent plus puissants que ceux prévalant à la poldérisation courante en Europe jusque dans les années 60 à 90 (BARON-YELLES \& GOELDNER-GIANELLA, 2001, GOELDNERGIANELLA, 2007). Ainsi, face aux submersions, le retour à la mer d'étendues inondables est bien moins couteux que le rehaussement des digues (DEFRA, 2002), de 


\section{Thème 6 - Gestion durable des zones littorales et estuariennes}

plus les zones dépoldérisées offrent un espace propice à la réduction des effets des tempêtes et des submersions, une protection pour les zones urbanisées proches (BAWEDIN \& HOEBLICH, 2006, BERTRAND \& GOELDNER-GIANELLA, 2011). Ce mode de gestion offre également des intérêts écologiques (BIELSTEIN et al., 1991), paysagers et patrimoniaux propices à l'accroissement de l'attractivité des sites. Ce sont ces moteurs de valorisation et d'ouverture au public, soutenus par une obligation de protection, qui ont poussé le Département de la Gironde à intégrer parmi ses outils de gestion des espaces naturels sensibles la renaturation, notamment par la voie de la dépoldérisation. L'île Nouvelle en est un exemple emblématique.

Née du fleuve il y a quelques centaines d'années, endiguée pour permettre le développement de l'agriculture, l'île Nouvelle, au cœur de l'estuaire de la Gironde (figure 1) fait aujourd'hui l'expérience particulièrement originale d'une dépoldérisation naturelle. En effet, suite à la rupture accidentelle d'une digue au cours de la tempête Xynthia, le secteur nord de l'île est le siège d'inondations tidales régulières qui guident des transformations morphologiques et biologiques très profondes. On note en particulier le creusement d'une brèche dans la digue, étirée dans l'île sous forme d'une coursive navigable, et la formation de vasières intertidales.

Un projet de recherche en cours vise à étudier les effets physiques, géochimiques, écologiques et socio-économiques de cette dépoldérisation accidentelle. La présente étude fait le bilan des observations menées dans le cadre de deux des volets de l'étude consacrés respectivement à l'évolution du couvert végétal et à la dynamique sédimentaire de la partie nord de l'île, ainsi que des analyses et premiers résultats proposés sur la base des données recueillies. Pour compléter ces résultats, et en particulier mieux comprendre l'impact de la dépoldérisation sur les ressources vivantes, nous proposerons une synthèse du volet de l'étude consacré à la macrofaune aquatique.

\section{Instrumentation, données et méthodes}

Deux approches ont été combinées. Des observations spatiales sont exploitées qui permettent de suivre l'évolution des zones humides (REBELO \& FINLAYSON, 2009), marais maritimes et zones intertidales (DEHOUCK et al., 2012), mais aussi de quantifier des indicateurs biogéophysiques qui lui sont propres par l'intermédiaire d'indices de végétation (GLENN et al., 2008). Les paramètres hydrosédimentaires permettant de comprendre les processus physiques guidant l'évolution de ce paysage sont appréhendés sur le terrain ainsi que les impacts sur la faune aquatique.

\subsection{Suivi du couvert végétal}

Dans le cas de l'île Nouvelle, les étapes de colonisation par de nouveaux cortèges floristiques ainsi que la transformation des sols sont observées par télédétection spatiale grâce à l'analyse de scènes satellites SPOT et Pléiades régulièrement acquises depuis 2009. Les images, projetées en Lambert93 et superposables, ont été traitées afin de 


\section{XIII ${ }^{\text {èmes }}$ Journées Nationales Génie Côtier - Génie Civil \\ Dunkerque, 2-4 juillet 2014}

caractériser l'évolution des surfaces occupées par une couverture végétale continue. Dans ce but, nous avons calculé l'indice de végétation normalisé (NDVI, ROUSE et al., 1973) de chaque image puis segmenté les image de NDVI sur la base d'un seuil discriminant les surfaces couvertes de végétation des sols nus. Ce seuil a été sélectionné afin d'exclure les zones couvertes de microphytobenthos, dont le NDVI est plus faible et la texture différente de ceux de la végétation enracinée sur laquelle nous nous concentrons. Les polygones extraits ont été vérifiés et validés individuellement. Pour interpréter correctement la carte produite, une mission d'observation a été réalisée le 10/10/2013 afin de caractériser les types de couverts de la zone dépoldérisée.
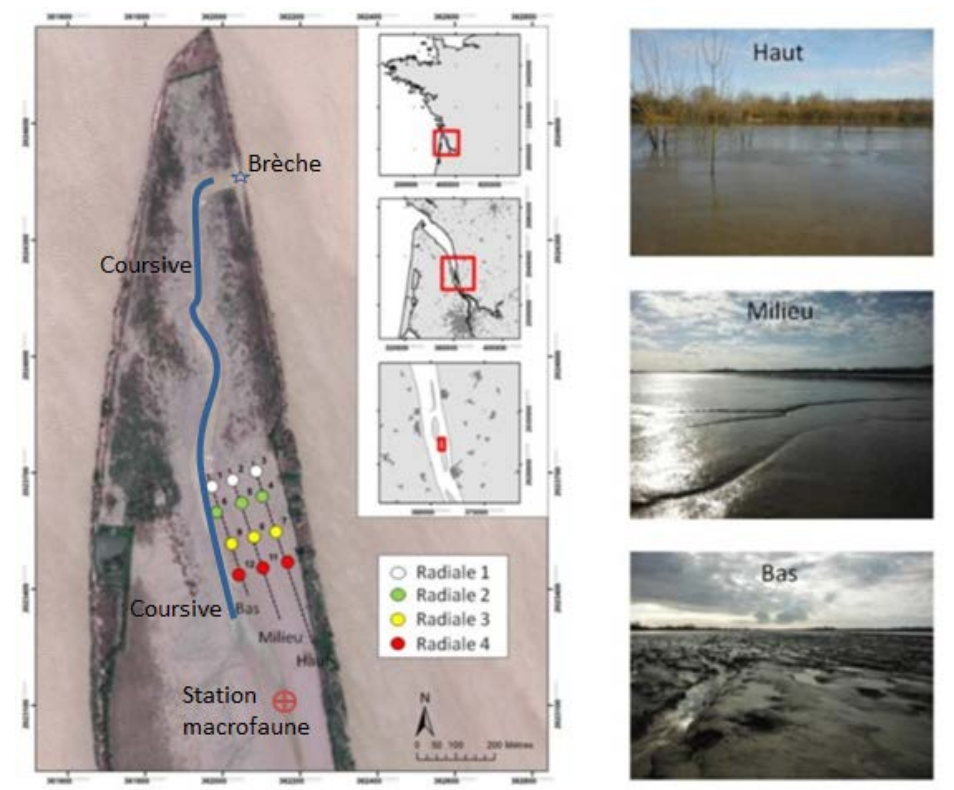

Figure 1. Localisation des stations de mesures le long des radiales dans la partie Nord de l'île Nouvelle et photos caractérisant les différents secteurs de la vasière orientale.

\subsection{Suivi morpho-sédimentaire}

Un suivi à moyen terme de l'évolution de la sédimentation de la vasière orientale a été réalisé à l'aide d'un dispositif constitué de jalons espacés de $50 \mathrm{~m}$ le long de quatre radiales transversales de $150 \mathrm{~m}$ de longueur (cf. figure 1). Ces jalons ont permis de mesurer, tous les mois entre septembre 2012 et mai 2013, la hauteur de l'interface sédimentaire, en fonction des forçages hydro-sédimentaires (débits de la Garonne et de la Dordogne, position du bouchon vaseux). Les mesures ont été réalisées lors de marées basses de vive-eau. La précision des mesures est en moyenne de $\pm 3 \mathrm{~mm}$. Ce suivi a été complété par des prélèvements de sédiments afin de caractériser la couverture sédimentaire.

Un suivi du contour de la coursive et de la brèche est également réalisé régulièrement depuis janvier 2011. Sept contours GPS (janvier 2001 - avril 2013) ont été réalisés par 


\section{Thème 6 - Gestion durable des zones littorales et estuariennes}

le Département de la Gironde. Un huitième a été obtenu par seuillage de l'image proche infrarouge de la scène Pléiades d'août 2013 qui révèle automatiquement l'interface entre l'eau et les surfaces émergées. Puis, le contour a été superposé à l’image panchromatique de Pléiades (50 cm de résolution) afin de vérifier le calage extrait.

\subsection{Suivi de la faune aquatique}

Les peuplements de poissons, crabes et crevettes ont été échantillonnés bimestriellement de mai 2011 à décembre 2012 (10 campagnes) afin de mettre en évidence les transformations de la faune aquatique consécutives à la dépoldérisation. Cinq stations de pêche ont été réparties sur l'ensemble de l'île : deux dans la partie nord (dépoldérisée) et trois dans la partie sud (endiguée). À chaque campagne et sur chaque station, deux modèles d'engins de capture passifs différant par leur maillage ont été déployés sur une durée de 24 heures. Les campagnes de pêche ont été réalisées pour des coefficients de marée compris entre 70 et 100 . Les poissons, crabes et crevettes capturés ont été identifiés à l'espèce, et, à défaut, au rang taxonomique supérieur le plus bas possible. Dans la mesure du possible, pour chaque taxon, les effectifs ont été dénombrés et leur biomasse totale a été mesurée directement sur le terrain avant que les individus capturés ne soient remis vivants dans leur milieu d'origine. Les poissons, crabes et crevettes ne pouvant être identifiés à l'espèce ont été sous-échantillonnés, congelés et examinés en laboratoire.

\section{Résultats}

\subsection{Suivi de la végétation}

Dans la zone contigüe à la brèche et la coursive, l'évolution de la couverture végétale est particulièrement importante. En 2011, la moitié des 63 ha initiaux est perdue au profit de vastes étendues envasées. En 2012, cette tendance s’atténue légèrement pour s'inverser en 2013 où on retrouve un recouvrement de près de 40 ha. Les images montrent que les zones situées à proximité de la brèche et de la coursive sont les plus affectées. Au contraire, les digues ont pu maintenir un couvert stable. Ces observations montrent que l'altitude est vraisemblablement un facteur majeur contrôlant la répartition spatiale du couvert végétal. La recolonisation progresse maintenant en direction de la coursive, sur des sols qui montrent dans le temps une évolution radiométrique très particulière, que l'on observe aussi bien au nord qu'au sud de la zone dépoldérisée (figure 2). Ainsi, à la place des anciens sols agricoles ou partiellement boisés (2009), s’étendent des sédiments fins de teneur en eau élevée (bien marquée dans la bande proche infrarouge, 2011). Un bassin a également été creusé à la faveur des marées. En 2012, le bassin est presque comblé. Au cours de l'été 2012 à certains endroits, la radiométrie indique que le sol acquiert de nouvelles propriétés : celles d'une surface très 


\section{XIII ${ }^{\text {èmes }}$ Journées Nationales Génie Côtier - Génie Civil \\ Dunkerque, 2-4 juillet 2014}

réfléchissante et très sèche (surface blanche sur l'image). La végétation y apparait enfin en 2013 sous forme de tâches plus ou moins vastes et espacées.
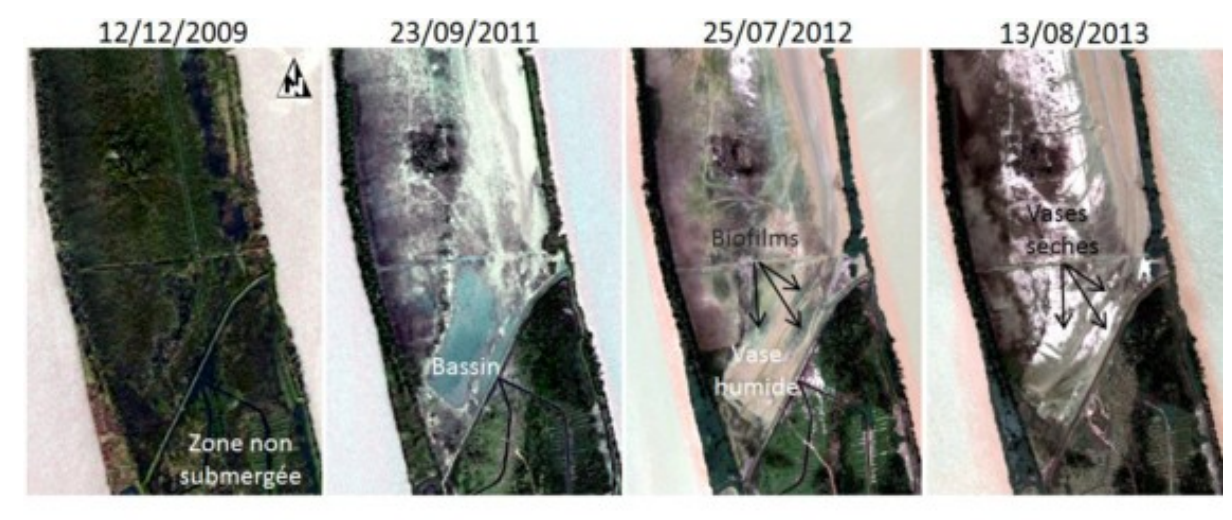

Figure 2. Evolution de la couverture végétale au nord de l'île Nouvelle entre 2009 et 2013(suivi estival).

Sur le terrain, on constate que les vases occupent les anciens bois de frênes et friches agricoles. Les vases sont étendues en couches plus ou moins nombreuses. En progressant depuis la coursive vers les digues, les espèces les plus communément rencontrées sont les phragmites et phalaris pour les herbacées associées parfois aux baccharis. Enfin des roselières occupent les digues. Par endroits, les phragmites colonisent les fentes de dessication ouvertes dans les vases asséchées qui ne sont sans doute uniquement atteintes que pour de hauts niveaux d'eau. Ainsi, la colonisation se produit sur les zones d'altitude propice. Cette colonisation favorise la stabilisation et la transformation du sol ce qui explique que l'on rencontre une colonisation primaire éparse se transformant en vastes étendues herbacées auxquelles succèdent et/ou se superposent des bouquets d'arbustes tolérants aux pressions environnementales. Toutes ces espèces coexistent dans le secteur méridional de l'île, endigué, attestant au nord la formation d'un marais maritime classique pour une île girondine.

\subsection{Suivi morpho-sédimentaire}

\subsubsection{Evolution de la brèche}

Un extrait des contours de la brèche et de la coursive dans l'île est présenté sur la figure 3. Entre l’ouverture de la brèche (fin février 2010) et le premier relevé (janvier 2011), la brèche ouverte dans la digue s'est propagée de $132 \mathrm{~m}$ vers l'ouest et $377 \mathrm{~m}$ vers le sud favorisant à la fois l'inondation et la vidange de l'île. Elle progresse ensuite vers le sud jusqu'en novembre 2012. Elle est depuis stabilisée, totalisant un déplacement de 2362 $m$ vers le sud. Dans le même temps, on observe également une légère érosion des deux rives de la coursive, l'érosion de la rive droite restant la plus marquée (10 m en moyenne depuis septembre 2011). 


\section{Thème 6 - Gestion durable des zones littorales et estuariennes}

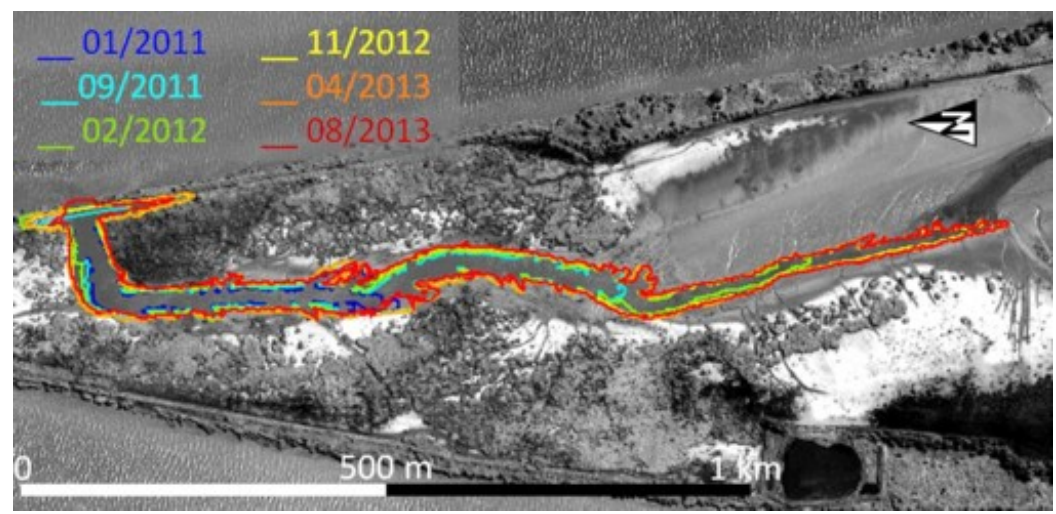

Figure 3. Evolution de la coursive ouverte dans l'île Nouvelle.

\subsubsection{Evolution de la sédimentation}

Les sédiments de surface constituant la vasière sont des vases silteuses ( $82 \%$ de silt), unimodales ( $8 \mu \mathrm{m}$ en moyenne) et globalement mal classées selon les critères de FOLK \& WARD (1957). La sédimentation observée suit une tendance similaire pour chacune des radiales indiquées sur la figure 1. En huit mois, les taux de sédimentation moyens sont compris entre $3,2 \mathrm{~cm} / \mathrm{mois}$ en haut de la vasière, $2,3 \mathrm{~cm} / \mathrm{mois}$ au milieu et $-0,025$ $\mathrm{cm} /$ mois pour les parties basses. Ces taux varient dans le temps. Ainsi, les plus forts envasements ont été observés en période d'étiage (débit de la Garonne et de la Dordogne cumulées $<500 \mathrm{~m}^{3} / \mathrm{s}$ ), lorsque le bouchon vaseux de la Gironde est positionné dans le secteur des îles. Entre le 22/10/2012 et le 29/11/2012, les dépôts de vase atteignent 8 à 12,6 cm sur les parties hautes, 5,3 à 6,7 cm sur les parties centrales et ils sont compris entre $-0,2$ et $+5,3 \mathrm{~cm}$ sur les parties basses de la vasière. En revanche, en période de crue, le bouchon vaseux se positionne plus en aval de ce secteur, les échanges entre la colonne d'eau chargée en MES et la vasière sont moins importants, provoquant une diminution notable de la sédimentation dans l'île Nouvelle. Ainsi, au cours de l'hiver 2012-2013 (débit de la Gironde $>1500 \mathrm{~m}^{3} / \mathrm{s}$ ), les envasements étaient plus faibles (en moyenne entre le 14/01/13 et le 18/02/13 : $-2 \mathrm{~cm}$ sur les parties basses, $+1,4 \mathrm{~cm}$ sur les parties centrales et $+2,5 \mathrm{~cm}$ sur les parties hautes). A partir du mois de mars 2013, l'envasement au milieu de la vasière augmente pour devenir équivalent à celui observé sur ses parties hautes. L'accumulation quotidienne de vase dans le haut de la vasière depuis mars 2010 fait que ce secteur n'est atteint que lors d'épisodes de viveeau ou de crues. Les eaux chargées en MES ont alors tendance à décanter de plus en plus longtemps sur les secteurs centraux de la vasière au détriment des parties hautes.

\subsection{Suivi de la faune aquatique}

Les effectifs et biomasses des peuplements de poissons et crustacés échantillonnés sur l'île Nouvelle sur la période 2011-2012 montrent que la dépoldérisation du secteur Nord de l'île Nouvelle s'accompagne d'une disparition ou d'une raréfaction très nette de plusieurs espèces de poissons d'eau douce comme le pseudorasbora, la gambusie et la 


\section{XIII ${ }^{\text {èmes }}$ Journées Nationales Génie Côtier - Génie Civil \\ Dunkerque, 2-4 juillet 2014}

carpe commune. L'abondance et la biomasse des épinoches décroissent très fortement. La partie dépoldérisée de l'île Nouvelle est massivement colonisée par le mulet porc, tant au niveau des effectifs que des biomasses, et dans une moindre mesure par le gobie tacheté. Les juvéniles de poissons marins (bar, anchois et sole), plus nombreux au nord qu'au sud, restent en effectifs limités. Les abondances de crevettes des marais déclinent dans la zone dépoldérisée avec une augmentation concomitante de l'importance de la crevette blanche Palaemon longirostris. Par rapport au secteur endigué, l'anguille reste fréquente et abondante dans la zone dépoldérisée.

\section{Synthèse et conclusions}

Les premiers résultats issus de l'analyse des données recueillies initient la compréhension de la mutation profonde qui s'opère sur l'ensemble du secteur septentrional de l'île Nouvelle, où l'envasement côtoie l'érosion depuis la rupture d'une digue entrainant la dépoldérisation de tout un territoire. La première étape, qui accompagne la submersion initiale, répétée ensuite au rythme des marées, implique 4 processus majeurs : une érosion très importante (brèche dans la digue et coursive vers l'intérieur des terres), la salinisation des sols, le déboisement et des apports de sédiments fins depuis le fleuve. Érosion et apports de sédiments se sont poursuivis lors des inondations tidales, clairement montrés par l'approfondissement et l'allongement de la coursive devenue navigable et les dépôts de sédiments fins notamment sous forme d'une vaste vasière au centre de l'île. Les sédiments s'accumulent au gré des marées et de la position du bouchon vaseux qui varie dans le temps, et on perçoit très rapidement les prémices d'une colonisation par différentes espèces classiques herbacées: phragmites, phalaris puis arbustives (baccharis). Passé un stade de colonisation primaire qui marque un comblement des zones de sédimentation, le couvert se densifie. Les espèces pionnières laissent rapidement place à une forte densification du couvert qui accompagne la stabilisation des zones où le comblement a été le plus actif. La colonisation végétale progresse rapidement remplaçant les anciens bois de frênes et friches agricoles en un couvert typique des marais estuariens. La sédimentation reste encore très active sur les sites de plus faible altitude. Des effets significatifs de cette transition sont également mis en évidence sur les assemblages de poissons et crustacés. On observe en effet une raréfaction très nette des espèces d'eau douce présentes dans les fossés avant dépoldérisation - carpe commune, gambusie, pseudorasbora - et l'apparition en abondance d'espèces estuariennes comme le mulet porc, le gobie tacheté, la crevette blanche $P$. longirostris et la crevette grise. La recolonisation du site par ces espèces indique un retour potentiel des fonctions d'alimentation et de refuge typiques des marais estuariens. Dans le contexte actuel de changement climatique et d'élévation du niveau de la mer, alors qu'un mouvement de rétrocession des polders à la mer se généralise en Europe occidentale, cette étude est un cas concret qui montre certains des impacts sédimentologiques et écologiques de la dépoldérisation en milieu estuarien. 
Thème 6 - Gestion durable des zones littorales et estuariennes

\section{Remerciements}

Nos remerciements vont au Département de la Gironde, au FUI (Programme INFOLITTORAL) et au CNES (programme ISIS).

\section{Références bibliographiques}

BAWEDIN V., HOEBLICH J.-M. (2006). Les Bas-Champs de Cayeux (Somme, France) : vers une gestion intégrée ? Enjeux et perspectives de l'ouverture à la mer d'un espace jusque-là protégé. Vertigo, Vol. 7, n³. http://dx.doi.org/10.4000/vertigo.1910

BARON-YELLES N., GOELDNER-GIANELLA L. (2001). Les marais maritimes d'Europe Atlantique, Paris, PUF, 294 p.

BERTRAND F., GOELDNER-GIANELLA L. (2011). Harmonisation des pratiques territoriales et des regards portés sur le risque de submersion marine dans le Basiin d'Arcachon : l'approche multi-scalaire du programme BARCASUB (MEEDTL, Liteau). Colloque international "Environnement et territoires : des représentations à l'action", Tours, 8 et 9 Décembre 2011.

BILDSTEIN K.L., BANCROFT G.T., DUGAN P.J, GORDON D.H., ERWIN R.M., NOL E., PAYNE L.X., SENNER S.E. (1991). Approaches to the conservation of coastal wetlands in the Western Hemisphere. Wilson Bull., Vol. 103(2), pp 218-254.

DEHOUCK A., LAFON V., LUBAC B., KERVELLA S., BRU D., SCHMELTZ M., ROUBACHE A. (2012). Hyperspectral field database in support to coastal wetland mapping. IGARSS 2012. IEEE International Geoscience and Remote Sensing Symposium, München, Germany, pp 2649-2652.

DEFRA -Environment Agency, Flood and Coastal Defence R\&D Programme- (2002). Managed Realignment Review, R\&D Technical Report FD2008, 212 p.

FOLK R.L., WARD W.C. (1957). Brazos River Bar: A study in the significance of grain size parameters. Journal of Sedimentary Petrology. Vol. 27, pp 3-26. http://dx.doi.org/10.1306/74D70646-2B21-11D7-8648000102C1865D

GLENN E.P., HUETE A.R., NAGLER P.L., NELSON S.G. (2008). Relationship Between Remotely-sensed Vegetation Indices, Canopy Attributes and Plant Physiological Processes: What Vegetation Indices Can and Cannot Tell Us About the Landscape. Sensors, Vol. 8, pp 2136-2160. http://dx.doi.org/10.3390/s8042136

GOELDNER-GIANELLA L. (2007). Dépoldériser en Europe. Annales de Géographie 2007/4, n 656, pp 339-360.

REBELO C.M., FINLAYSON N.N. (2009). Remote sensing and GIS for wetland inventory, mapping and change analysis. J. Environ. Manag., Vol. 90, pp 2144-2153. http://dx.doi.org/10.1016/j.jenvman.2007.06.027

ROUSE J.W., HAAS R.H., SCHELL J. A., DEERING D. W. (1973). Monitoring vegetation systems in the Great Plains with ERTS. Third ERTS Symposium, NASA SP351 I, pp 309-317. 